\title{
A model of magnetic stimulation of neocortical neurons
}

\author{
Yukiyasu Kamitania,*, Vidya M. Bhalodia ${ }^{\mathrm{b}}$, Yoshihisa Kubota ${ }^{\mathrm{c}}$, \\ Shinsuke Shimojo ${ }^{\mathrm{a}, \mathrm{d}}$ \\ ${ }^{\mathrm{a} C}$ Computation and Neural Systems, California Institute of Technology, MC 139-74, Pasadena, CA, USA \\ ${ }^{\mathrm{b}}$ Department of Neuroscience, Washington University in St. Louis, Campus Box 8226, St. Louis, MO 63110, USA \\ ${ }^{\mathrm{c} C o n t r o l}$ and Dynamical Systems, California Institute of Technology, MC 107-81, Pasadena, CA, USA \\ ${ }^{\mathrm{d}}$ Human and Information Science Laboratory, NTT Communication Science Laboratories, Atsugi, \\ Kanagawa 243-0198, Japan
}

\begin{abstract}
Transcranial magnetic stimulation (TMS) has been widely used in studies of human motor and cognitive functions as well as in clinical treatment. Biophysical mechanism underlying its effect is, however, largely unknown. Here, we develop a theory to calculate the effect of magnetic stimulation on arbitrary neuronal structure. Then, we employ a computer simulation which combines a realistic multicompartmental model of neocortical neurons and the calculation of the induced electric field. The simulation shows that a single magnetic pulse applied to model cortical neurons can induce brief burst firing followed by a silent period of duration comparable to experimental data of TMS. Our simulation offers a new clue to understand physiology of TMS by demonstrating that magnetic stimulation acts on biophysics of the dendrites in neocortical neurons. (C) 2001 Elsevier Science B.V. All rights reserved.
\end{abstract}

Keywords: Magnetic stimulation; Neocortical neuron; Dendrite; Compartmental model

\section{Introduction}

Transcranial magnetic stimulation (TMS), a non-invasive technique to stimulate the human cerebral cortex by a brief magnetic pulse through a coil put on the scalp [2], has been widely used in studies of human motor and cognitive functions as well as in clinical treatment. Biophysical mechanism underlying its effect is, however, largely

\footnotetext{
* Corresponding author.

E-mail address: kamitani@caltech.edu (Y. Kamitani).
} 
unknown. Previous theoretical studies $[10,8]$ have demonstrated that a brief magnetic pulse (less than $1 \mathrm{~ms}$ duration), commonly used in TMS, can generate an action potential in a straight axonal fiber. Nevertheless, TMS typically demonstrates effects including a long inhibitory period (a few hundred ms) as observed in EMG [3] and visual perception $[1,5]$.

In this study, we develop a theory to calculate the effect of magnetic stimulation on arbitrary neuronal structure. Then, we employ a computer simulation which combines a realistic multicompartmental model of neocortical neurons and the calculation of the induced electric field. We demonstrate that a single magnetic pulse applied to model cortical neurons can induce brief burst firing followed by a silent period of duration comparable to experimental data of TMS.

\section{Methods}

\subsection{Simulation of magnetic stimulation}

TMS exerts its effect on cortical neurons through a mechanism different from its action on a straight axonal fiber in which transmembrane current is induced by the spatial gradient of induced electric field [10]. For cortical neurons, the induced electric field is nearly uniform because of the small size of neurons as compared to the size of coils used in TMS. Instead, branching and change of size and orientation in dendritic tree do play a significant role. The derivation of induced transmembrane current is as follows.

A magnetic pulse induces an electric field given by

$$
\mathbf{E}=\frac{\partial \mathbf{A}}{\partial t}
$$

where $\mathbf{A}$ is the vector potential of the magnetic field produced by the coil. The magnetic stimulation results in axial current induction inside the fiber given by

$$
I_{\mathrm{ind}}=\frac{E_{i}}{r_{i}}
$$

where $r_{i}$ is the axial resistance of the fiber and $E_{i}$ is the axial component of the electric field. Thus, the density of additional transmembrane current induced by magnetic stimulation is derived by taking spatial derivative of the above axial current $I_{\text {ind }}$

$$
I_{\mathrm{tr}}-\frac{\partial}{\partial s}\left(\frac{E_{i}(s)}{r_{i}(s)}\right)
$$

where $s$ is the position along the fiber of interest. As noted from this formula, change in diameter (determining axial resistance) and direction of the fiber as well as the spatial gradient of the electric field gives rise to transmembrane current induction. In our 
simulation, the transmembrane current induced at each compartment is calculated by

$$
I_{\text {comp }}=\int_{s_{0}}^{s_{1}}-\frac{\partial}{\partial s}\left(\frac{E_{i}(s)}{r_{i}(s)}\right) \mathrm{d} s=\frac{E_{i}\left(s_{0}\right)}{r_{i}\left(s_{0}\right)}-\frac{E_{i}\left(s_{1}\right)}{r_{i}\left(s_{1}\right)}
$$

where $s_{0}$ and $s_{1}$ are the two ends of the compartment. Note that the total transmembrane current in a compartment is determined only by the induced currents at the two ends, regardless of those between these points. This transmembrane current was injected in a multicompartmental model to simulate the effect of magnetic stimulation (Fig. 1a).

Furthermore, as pointed out by Nagarajan et al. [8], at the neuronal terminals where the induced current flows toward (away from) them, magnetic stimulation results in the equivalent amount of current injection (subtraction) (Fig. 1b). Also, at the branching points, magnetic stimulation leads to current injection due to the difference between the induced current in a parent fiber and the sum of those in the branching fibers (Fig. 1c).

(a)
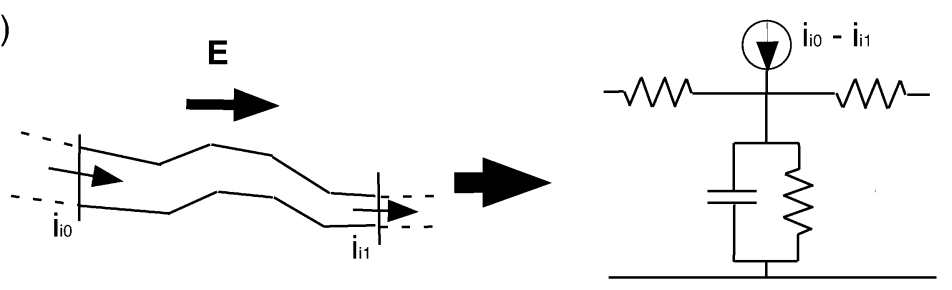

(b)
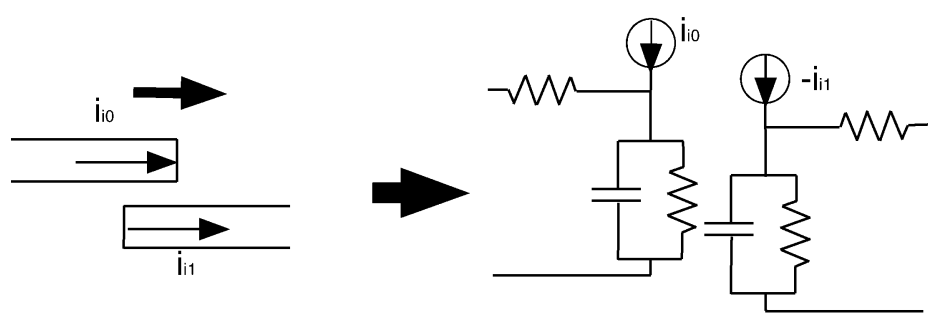

(c)
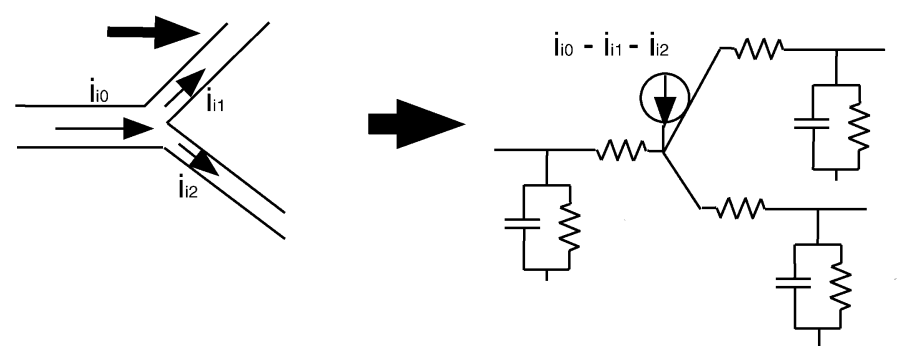

Fig. 1. Implementation of magnetic stimulation in compartmental models. The effect of magnetic stimulation on parts of neurons (left column) and the implementation in compartmental models (right column) are shown for a segment (a), ends (b), and a branching point (c). $\mathbf{E}$ is the electric field induced by magnetic stimulation. $i_{i 0-2}$ are the induced currents in the fibers, which are determined by the axial component of the induced electric field $\mathbf{E}$ and the axial resistance of the fiber. 
Interestingly, the above theory deduced that the total amount of current injected into an entire neuron is zero at any moment. This implies that in neurons of very short electronic length, positive and negative injected currents are likely to be canceled out without causing any significant effects on the local membrane potential.

The magnetic stimulator and the coil were simulated as an LRC circuit, and all parameters were adjusted to a commercial magnetic stimulator Magstim200 (The Magstim Company Ltd, UK). The maximal output was set to about $500 \mathrm{~V} / \mathrm{m}$ and the pulse duration was less than $1 \mathrm{~ms}$.

\subsection{Multicompartmental model of cortical neuron}

The neocortical neuron model was taken and modified from Mainen and Sejnowski [7]. Briefly, the model neurons had a low density of $\mathrm{Na}^{+}$channels in the soma and dendrites, and a high density in the axon hillock and initial segment. Fast $\mathrm{K}^{+}$channels were present in the axon and soma, while slow calcium- and voltagedependent $\mathrm{K}^{+}$channels were in the soma and dendrites. High threshold $\mathrm{Ca}^{2+}$ channels were present in soma and dendrites. Dendritic arborizations were reconstructed from the literature. We also analyzed the effect of background synaptic inputs. We modeled excitatory synapses randomly distributed on the dendrites, and a Poisson distribution for EPSPs trains. Simulations were performed under the NEURON [4] environment.

\section{Summary of simulation results}

1. A magnetic pulse produced a long inhibitory period following a brief bursting in a Layer 5 pyramidal cell model (Fig. 2). The threshold of magnetic stimulation was lower when background inputs were present.

2. It was difficult to cause the long dynamics in cell models with small neuronal size such as a Layer 4 spiny stellate cell as predicted.

3. For the Layer 5 pyramidal cell, the electric fields with a direction parallel to the major trunk of the apical dendrites were more effective than orthogonal ones.

4. Our analysis revealed that $\mathrm{Ca}^{2+}$ influx followed by the opening of calciumdependent $\mathrm{K}^{+}$channels is responsible for the bursting followed by the long hyperpolarization period.

\section{Conclusion and physiological implication}

To summarize, our realistic neuronal model predicted burst firing followed by a long silent period of neuronal activity (when background synaptic inputs are present) similar in time course to those observed in behavioral and cognitive experiments using TMS. The higher sensitivity to magnetic stimulation under background synaptic inputs is also consistent with the fact that the effects of TMS are enhanced during muscle contraction or intense sensory input [3,5]. Intracellular $\mathrm{Ca}^{2+}$ is known 


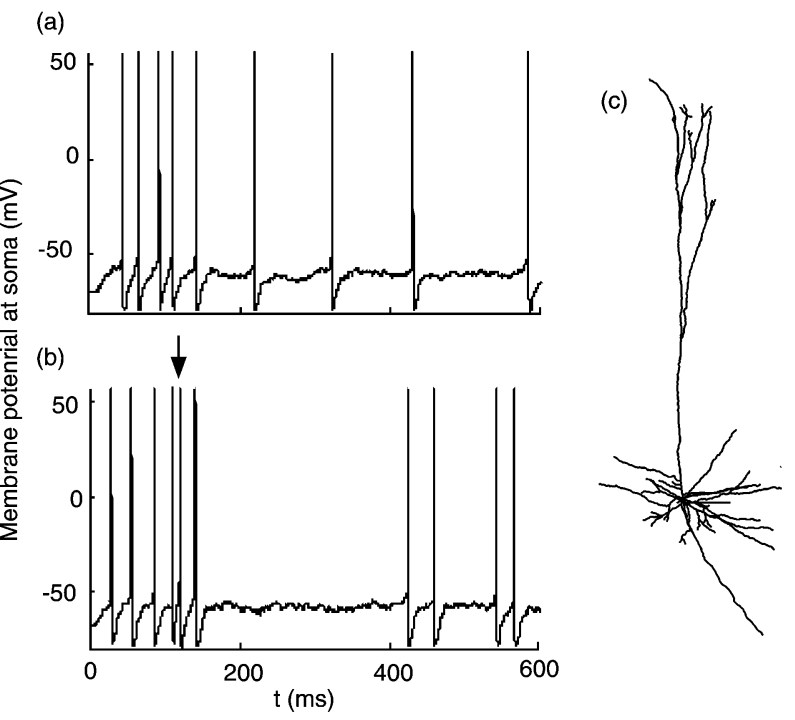

Fig. 2. Simulation results. (a) Membrane potential at the soma under background synaptic inputs, with no magnetic stimulation. (b) The same model as (a) except that a magnetic pulse was delivered at the timing indicated by the arrow. (c) Microscopically reconstructed neuronal structure (Layer 5 pyramidal cell) used in this simulation.

to lead to persistent change in neuronal function. Therefore, the massive dendritic $\mathrm{Ca}^{2+}$ influx induced by magnetic stimulation may underlie TMS-induced long-term effects on cognitive performance [6], and mood in depression patients [9].

\section{References}

[1] V.E. Amassian, R.Q. Cracco, P.J. Maccabee, J.B. Cracco, A. Rudell, L. Eberle, Suppression of visual perception by magnetic coil stimulation of human occipital cortex, Electroencephalogr. Clin. Neurophysiol. 74 (1989) 458-462.

[2] A.T. Barker, R. Jalinous, I.L. Freeston, Non-invasive magnetic stimulation of human motor cortex, Lancet 1 (1985) 1106-1107.

[3] M. Hallet, Transcranial magnetic stimulation: negative effects, in: S. Fahn, M. Hallet, H.O. Lübers, C.D. Marsden (Eds.), Negative Motor Phenomena, Lippincott-Raven Publishers, Philadelphia, 1995.

[4] M. Hines, NEURON: a program for simulation of nerve equations, in: F. Eeckman (Ed.), Neural Systems: Analysis and Modeling, Kluwer Academic Publishers, Boston, MA, 1993, pp. 127-136.

[5] Y. Kamitani, S. Shimojo, Manifestation of scotomas created by transcranial magnetic stimulation of human visual cortex, Nat. Neurosci. 2 (1999) 767-771.

[6] S.M. Kosslyn, A. Pascual-Leone, O. Felician, S. Camposano, J.P. Keenan, W.L. Thompson, G. Ganis, K.E. Sukel, N.M. Alpert, The role of Area 17 in visual imagery: convergent evidence from PET and rTMS, Science 284 (1999) 167-170. 
[7] Z.F. Mainen, T.J. Sejnowski, Influence of dendritic structure on firing pattern in model neocortical neurons, Nature 382 (1996) 363-366.

[8] S.S. Nagarajan, D.M. Durand, E. Warman, Effects of induced electric fields on finite neuronal structures: a simulation study, IEEE Trans. Biomed. Eng. 40 (1993) 1175-1188.

[9] A. Pascual-Leone, B. Rubio, F. Pallardo, M.D. Catala, Rapid-rate transcranial magnetic stimulation of left dorsolateral prefrontal cortex in drug-resistant depression, Lancet 348 (1996) 233-237.

[10] B.J. Roth, P.J. Basser, A model of the stimulation of a nerve fiber by electromagnetic induction, IEEE Trans. Biomed. Eng. 37 (1990) 588-597.

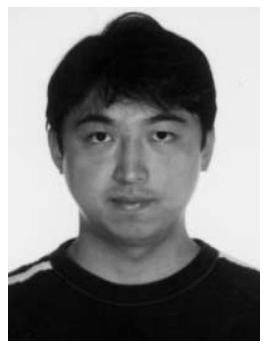

Yukiyasu Kamitani is at Computation and Neural Systems Program at California Institute of Technology. His main focus of research is on theory and application of transcranial magnetic stimulation (TMS). He works on computational modeling of single neurons under magnetic stimulation. He has also studied effects of TMS on the human occipital cortex in visual perception. Combining these, he aims to study the relationship between neural electrical activity and conscious perceptual experience. Other interests include psychophysics of visual perception and auditory-visual integration.

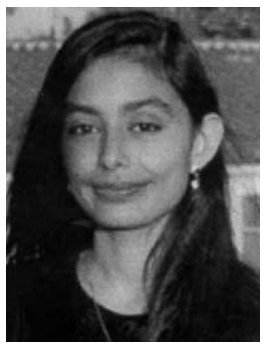

Vidya M. Bhalodia is currently pursuing her Ph.D. in Neuroscience at Washington University in St. Louis, MO. She obtained her B.S. in Biology from California Institute of Technology, Pasadena, CA.

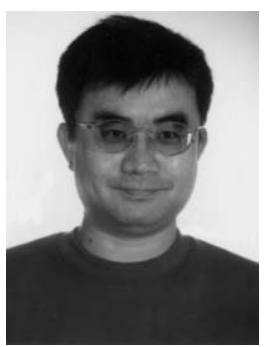

Dr. Kubota is at Control and Dynamical Systems, and Division of Biology at Caltech. His scientific research is currently focused on how molecular protein machinery in synapses of mammalian nervous system is synchronously organized and works together in processing fine time-scale temporal structure of neuronal signals under noisy environment. Various applied and computational mathematical methods are employed to explore the molecular control mechanism of the phenomena. On the experimental side, he is organizing a collaboration with biochemists to test predictions made by the modeling approach. Nonlinear system analysis in computational neuroscience, especially nonlinear cable theory of dendrite, is another area of his research effort which results in joint collaboration on TMS simulation with realistic neuronal model. 


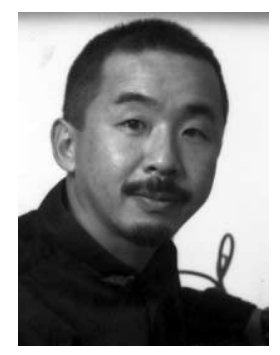

Dr. Shimojo is a professor in Division of Biology and Computation and Neural Systems at Caltech. He is also an adjunct research professor at NTT Communication Science Laboratories. His psychophysical laboratory at Caltech is devoted for a variety of issues in cognitive neurosciences, including: (a) the causal relationship between visual cortical activity and subjective experience of perception, assessed by TMS (transcranial magnetic stimulation), (b) auditory-visual integration and its development in the human infants, (c) perception of simultaneity and temporal-order among cross-modal sensory events, (d) localization of transient visual stimulus with regard to motion stimulus, (e) fMRI study of brain mechanisms for dynamic integration of visual contour, (f) pupil and MEG measures of response to color flicker in normal and photo-sensitive humans, $(\mathrm{g})$ perception and short-term memory of space, and (h) relationship among memory, emotion and orienting behavior. 